Konrad-Zuse-Zentrum für Informationstechnik Berlin

Heilbronner Str. 10, D-1000 Berlin 31

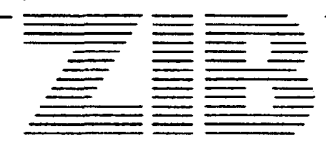

Peter Deufhard Roland Freund * Artur Walter

\author{
Fast Secant Methods \\ for the Iterative Solution \\ of Large Nonsymmetric Linear Systems
}

RIACS, Mail Stop T045-1

NASA Ames Research Center

Moffett Field, CA 94035

U.S.A.

The research of this author was supported in part

by DARPA via Cooperative Agreement NCC 2-387 between

NASA and the Universities Space Research Association (USRA).

Preprint SC 90-5 (July 1990) 
Peter Deuflhard Roland Freund Artur Walter

\title{
Fast Secant Methods for the Iterative Solution of Large Nonsymmetric Linear Systems
}

\begin{abstract}
A family of secant methods based on general rank-1 updates has been revisited in view of the construction of iterative solvers for large non-Hermitian linear systems. As it turns out, both Broyden's "good" and "bad" update techniques play a special role - but should be associated with two different line search principles. For Broyden's "bad" update technique, a minimum residual principle is natural - thus making it theoretically comparable with a series of well-known algorithms like GMRES. Broyden's "good" update technique, however, is shown to be naturally linked with a minimum "next correction" principle - which asymptotically mimics a minimum error principle. The two minimization principles differ significantly for sufficiently large system dimension. Numerical experiments on discretized PDE's of convection diffusion type in 2-D with internal layers give a first impression of the possible power of the derived "good" Broyden variant.
\end{abstract}

Key Words: nonsymmetric linear system, secant method, rank-1 update, Broyden's method, line search, GMRES.

AMS(MOS) Subject Classifications: 65F10, 65N20 
The authors would like to thank Mrs. Erlinda Cadano-Körnig for her excellent $\mathrm{T}_{\mathrm{EX}} \mathrm{X}$-typing of the manuscript. 


\section{Contents}

1. Introduction 1

2. A Family of Secant Methods 3

2.1 The General Algorithm . . . . . . . . . . . . . . . 3

2.2 Special Rank-1 Updates . . . . . . . . . . . . . . . . . . . . . .

2.3 Line Search Principles . . . . . . . . . . . . . 8

3. Convergence Analysis $\quad 12$

3.1 Broyden's Bad Update . . . . . . . . . . . . . . . . . . . . 12

3.2 Broyden's Good Update . . . . . . . . . . . . . . . 14

3.3 An Illustrative Example . . . . . . . . . . . . . 17

4. Details of Realization $\quad 21$

5. Numerical Experiments $\quad 26$

$\begin{array}{ll}\text { Conclusion } & 32\end{array}$

$\begin{array}{ll}\text { References } & 33\end{array}$ 


\section{Introduction}

The solution of large sparse systems of linear equations

$$
A x=b
$$

is one of the most frequently encountered tasks in numerical computations. In particular, such systems arise from finite difference or finite element approximations to partial differential equations (PDEs). For Hermitian positive definite coefficient matrices $A$, the classical conjugate gradient method (CG) of HESTENES/STIEFEL [11] is one of the most powerful iterative techniques for solving (1.1).

In recent years, a number of CG type methods for solving general non-Hermitian linear systems (1.1) have been proposed. The most widely used of these algorithms is GMRES due to SAAD/SCHULTZ [13]. However, solving non-Hermitian linear systems is, in general, by far more difficult than the case of Hermitian $A$, and the situation is still not very satisfactory. For instance, this is reflected in the fact that for methods such as GMRES work and storage per iteration grow linearly with the iteration number $k$. Consequently, in practice, one can not afford to run the full algorithm and restarted or truncated versions are used instead. Notice that, on the contrary, CG for Hermitian $A$ is based on a three-term recursion and thus work and storage per iteration remain constant.

Non-Hermitian linear systems (1.1) are special cases of systems of nonlinear equations. For sufficiently good initial guesses, secant methods (see e.g. DENNIS/SCHNABEL [3]) based on Broyden's rank-1 updates are known to be quite efficient techniques for solving these more general problems. However, up to now, secant methods for solving linear systems have had a bad reputation.

The purpose of this paper is to take an unusual look at secant methods for non-Hermitian linear systems (1.1). In particular, as will be shown, combining Broyden's good and bad updates with different line search principles leads to iterative schemes which are competitive with GMRES. More than that, these secant methods typically exhibit a better reduction of the Euclidean error than GMRES. This is of particular importance for solving linear systems which arise in the context of multilevel discretizations of PDEs. There, linear systems are only solved to an accuracy corresponding to the discretization error on the respective level. In order to obtain such approximate solutions with as few iterations as possible, reduction of the Euclidean error is typically more crucial than minimizing the residual norm as GMRES does. For a description of such multilevel techniques, see the recent paper of DEUFLHARD/LEINEN/YSERENTANT $[5]$. 


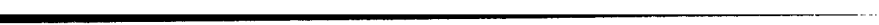


It is well known (see e.g. Fletcher [7, Chapter 3]) that CG for Hermitian positive definite $A$ is intimately connected with minimization algorithms based on Broyden's family of rank-2 updates. In view of this result, the similar behavior of GMRES and secant methods based on rank-1 updates might not come as a surprise. Nevertheless, there appears to be no strict connection between the two techniques. Recently, however, EIrola/NEVANLINna [6] have established a connection between GMRES and a certain rank-1 update based on a nonstandard secant condition (cf. Remark 1 in Section 2.1).

The paper is organized as follows. In Section 2.1, we introduce a general family of secant methods. In Sections 2.2 and 2.3, special rank-1 updates and line search principles, respectively, are discussed. In Sections 3.1 and 3.2, we present convergence results for secant methods based on Broyden's bad and good updates. These results are then illustrated for a linear system arising from a simple 1-D boundary value problem in Section 3.3. Next, we discuss actual implementations of the proposed secant methods in Section 4. Typical numerical experiments are reported in Section 5. Finally, we make some concluding remarks.

Throughout this paper, all vectors and matrices are assumed to be complex. As usual, $M^{*}=\left(\overline{m_{k j}}\right)$ denotes the conjugate transpose of the matrix $M=$ $\left(m_{j k}\right)$. The vector norm $\|x\|=\sqrt{x^{*} x}$ is always the Euclidean norm and $\|M\|=$ $\sup _{\|x\|=1}\|M x\|$ the corresponding matrix norm. Occasionally, the Frobenius norm $\|M\|_{F}=\left(\sum_{j, k}\left|m_{j k}\right|^{2}\right)^{1 / 2}$ will be used. 


\section{A Family of Secant Methods}

The paper deals with the solution of linear systems (1.1) where $A$ is a nonHermitian $n \times n$ matrix and $b \in \mathbb{C}^{n}$. From now on, it is always assumed that $A$ is nonsingular, and $x:=A^{-1} b$ denotes the exact solution of (1.1).

The methods studied in this paper are iterative schemes. For any given starting vector $x_{0} \in \mathbb{C}^{n}$, a sequence of approximations $x_{k}, k=1,2, \ldots$, to $x$ is computed. Furthermore, in each step an $n \times n$ matrix $H_{k}$ which approximates $A^{-1}$ is generated. Here $H_{0}$ is a given nonsingular initial approximation of $A^{-1}$.

In the sequel,

$$
e_{k}:=x-x_{k} \text { and } r_{k}:=b-A x_{k}
$$

always denote the error vector and residual vector, respectively, corresponding to the iterate $x_{k}$. Moreover,

$$
E_{k}:=I-H_{k} A
$$

is the error matrix associated with the "preconditioning" matrix $H_{k}$ and

$$
\Delta_{k}:=H_{k} r_{k}
$$

is the "preconditioned" residual vector. Finally, for nonsingular $H_{k}$, we denote by

$$
B_{k}:=H_{k}^{-1}
$$

the approximations of $A$.

\subsection{The General Algorithm}

The approximation $H_{k+1}$ of $A$ is obtained from the one of the previous iteration, $H_{k}$, by adding a rank-1 correction. In conjunction with the requirement that the following secant condition (or quasi-Newton condition)

$$
H_{k+1} A \Delta_{k}=\Delta_{k}
$$

holds, this leads (see e.g. [3, Chapter 8]) to the general update

$$
H_{k+1}=H_{k}+\left(I-H_{k} A\right) \frac{\Delta_{k} v_{k}^{*}}{v_{k}^{*} H_{k} A \Delta_{k}} H_{k}
$$

due to Broyden [1]. Here, $v_{k} \in \mathbb{C}^{n}$ is any vector such that $v_{k}^{*} H_{k} A \Delta_{k} \neq 0$. By applying the Sherman-Morrison formula to (2.2), one readily verifies that $H_{k+1}$ is nonsingular with inverse

$$
B_{k+1}=B_{k}+\left(A-B_{k}\right) \frac{\Delta_{k} v_{k}^{*}}{v_{k}^{*} \Delta_{k}}
$$


as long as $H_{k}$ is nonsingular and $v_{k}^{*} \Delta_{k} \neq 0$.

Remark 1. Eirola/Nevanlinna [6] study secant methods which are based on the "conjugate transposed" secant condition

$$
H_{k+1}^{*} A^{*} c_{k}=c_{k}
$$

instead of (2.1). For the special choice $c_{k}=A \Delta_{k}$ in (2.4), the resulting algorithm ([6], see also [14]) is mathematically equivalent to GMRES.

In each iteration, the new approximation $x_{k+1}$ to $x$ is obtained by correcting the previous iterate $x_{k}$ along the preconditioned residual $\Delta_{k}$. In combination with the update (2.2), this leads to the following informal algorithm.

\section{Algorithm 2.1}

$$
\begin{aligned}
& \text { Start: a) } r_{0}:=b-A x_{0} \\
& \text { Iteration loop: } k=0,1, \ldots: \\
& \text { b) } \Delta_{k}:=H_{k} r_{k} \\
& q_{k}:=A \Delta_{k} \\
& z_{k}:=H_{k} q_{k} \\
& \text { c) } x_{k+1}:=x_{k}+t_{k} \Delta_{k} \\
& r_{k+1}:=r_{k}-t_{k} q_{k}
\end{aligned}
$$

Update:

$$
\text { d) } H_{k+1}:=H_{k}+\left(\Delta_{k}-z_{k}\right) \frac{v_{k}^{*} H_{k}}{v_{k}^{*} z_{k}}
$$

Notice that Algorithm 2.1 describes a whole family of secant methods which still depend on the choices of $v_{k}$ in the update d) and the step length $t_{k}$ in c). Strategies for the selection of these parameters will be discussed in Sections 2.2 and 2.3.

In the following lemma, we collect some simple recursions that are valid for all choices of $v_{k}$ and $t_{k}$. Here and in the sequel, the notations

$$
\tau_{k}:=\frac{v_{k}^{*} \Delta_{k}}{v_{k}^{*} z_{k}}, \quad \widetilde{z}_{i}=\widetilde{z}_{i}^{(k)}:=H_{i} A \Delta_{k}, \quad \text { and } \quad \gamma_{i}:=\frac{v_{i}^{*} \widetilde{z}_{i}}{v_{i}^{*} z_{i}}
$$

are used. 
Lemma 2.2 Let $v_{i}^{*} z_{i} \neq 0, i=0, \ldots, k-1$. Then:

$$
\begin{aligned}
& \text { a) } e_{k+1}=\left(\left(1-t_{k}\right) I+t_{k} E_{k}\right) e_{k} \\
& \text { b) } \left.\Delta_{k+1}=\left(1-t_{k}+\tau_{k}\right) \Delta_{k}-\tau_{k} z_{k}\right)=\left(\left(1-t_{k}\right) I+\tau_{k} E_{k}\right) \Delta_{k} \\
& \text { c) } \tilde{z}_{i+1}=\tilde{z}_{i}+\frac{\gamma_{i}}{\tau_{i}}\left(\Delta_{i+1}-\left(1-t_{i}\right) \Delta_{i}\right), i=0, \ldots, k-1
\end{aligned}
$$

Proof. Note that $e_{k+1}=e_{k}-t_{k} \Delta_{k}$ and $\Delta_{k}=\left(I-E_{k}\right) e_{k}$. Combining these two identities yields $(2.6 \mathrm{a})$.

Next, one easily verifies that

$$
\Delta_{k+1}=H_{k+1} r_{k+1}=\left(1-t_{k}\right) \Delta_{k}+\tau_{k}\left(\Delta_{k}-z_{k}\right) .
$$

Since $E_{k} \Delta_{k}=\Delta_{k}-z_{k},(2.7)$ immediately leads to (2.6b).

By using (2.5) and the update formula which connects $H_{i+1}$ and $H_{i}$, one obtains

$$
\tilde{z}_{i+1}=\tilde{z}_{i}+\gamma_{i}\left(\Delta_{i}-z_{i}\right)
$$

Finally, by rewriting the term $\Delta_{i}-z_{i}$ in (2.8) by means of the first identity (with $k=i$ ) in $(2.6 \mathrm{~b})$, one arrives at $(2.6 \mathrm{c})$.

\subsection{Special Rank-1 Updates}

First, note that, by (2.2), the error matrix associated with the preconditioner $H_{k}$ satisfies the update formula

$$
E_{k+1}=E_{k}\left(I-\frac{\Delta_{k} v_{k}^{*}}{v_{k}^{*} z_{k}} H_{k} A\right) .
$$

Clearly, one would like to improve the preconditioner from step to step. Thus, $v_{k}$ in (2.9) should be chosen such that a suitable norm of $E_{k}$ is decreasing. In this section, three special choices of $v_{k}$ are discussed.

(A) The first one is the so-called Broyden's "good" update [1]. Here, in each iteration, one sets

$$
v_{k}:=\Delta_{k} .
$$

Assume that $H_{k}$ is nonsingular and that $x_{k} \neq x$, which implies $\Delta_{k} \neq 0$. With (2.10), (2.9) can be rewritten as

$$
E_{k+1}=E_{k} P_{k} \quad \text { where } \quad P_{k}:=\left(I-\frac{\Delta_{k} \Delta_{k}^{*}}{\Delta_{k}^{*} H_{k} A \Delta_{k}} H_{k} A\right) .
$$


Remark that, except for the trivial case $H_{k} A=I, P_{k}$ in (2.11) is an oblique, non-orthogonal projection. Thus, one cannot guarantee that $\left\|E_{k}\right\|$ is decreasing. However, for the different error matrix

$$
\widetilde{E}_{k}:=I-A^{-1} B_{k},
$$

one obtains such a reduction property:

$$
\tilde{E}_{k+1}=\tilde{E}_{k} Q_{k} \text { where } Q_{k}:=\left(I-\frac{\Delta_{k} \Delta_{k}^{*}}{\Delta_{k}^{*} \Delta_{k}}\right) .
$$

Now, $Q_{k}$ is an orthogonal projection. Consequently, (2.12) guarantees an improvement of the preconditioner in each step, in the sense that

$$
\left\|\tilde{E}_{k+1}\right\| \leq\left\|\tilde{E}_{k}\right\|
$$

and

$$
\left\|\tilde{E}_{k+1}\right\|_{F}^{2}=\left\|\tilde{E}_{k}\right\|_{F}^{2}-\frac{\left\|\tilde{E}_{k} \Delta_{k}\right\|^{2}}{\left\|\Delta_{k}\right\|^{2}} .
$$

Obviously, in view of (2.2) and (2.10), Broyden's good update is only defined as long as

$$
\Delta_{k}^{*} H_{k} A \Delta_{k} \neq 0
$$

which (cf. (2.3)) guarantees that with $H_{k}$ also $H_{k+1}$ is nonsingular.

In particular, the more restrictive condition

$$
\Delta_{k}^{*} H_{k} A \Delta_{k}>0
$$

certainly implies (2.15). Clearly, (2.16) can be rewritten as

$$
\varepsilon_{k}:=\frac{\Delta_{k}^{*} E_{k} \Delta_{k}}{\Delta_{k}^{*} \Delta_{k}}<1
$$

Since $\varepsilon_{k} \leq\left\|E_{k}\right\|$, a sufficient condition for (2.17) is

$$
\left\|E_{k}\right\|<1 \text {. }
$$

Now, it is easily verified that $\widetilde{E}_{k}$ and $E_{k}$ are connected by

$$
E_{k}=-\left(I-\widetilde{E}_{k}\right)^{-1} \tilde{E}_{k},
$$

and it follows that

$$
\left\|E_{k}\right\| \leq \frac{\left\|\widetilde{E}_{k}\right\|}{1-\left\|\widetilde{E}_{k}\right\|}
$$


By (2.19), the condition

$$
\left\|\widetilde{E}_{k}\right\|<\frac{1}{2}
$$

implies (2.18). If (2.20) is satisfied for $k=0$, then (2.13) guarantees that (2.20) holds for all $k$. Finally, by (2.18), $H_{k} A$ and, since $A$ is assumed to be nonsingular, $H_{k}$ is nonsingular.

Therefore, we have proved the following

Lemma 2.3 Let $H_{0}$ be a nonsingular $n \times n$ matrix such that $\left\|\widetilde{E}_{0}\right\|<\frac{1}{2}$. Then, Broyden's good update (2.2), with $v_{k}$ chosen as in (2.10), is well defined as long as $x_{k} \neq x$.

(B) The so-called Broyden's "bad" update [1] is obtained by choosing $v_{k}$ in (2.2) such that

$$
H_{k}^{*} v_{k}=A \Delta_{k}=q_{k}
$$

holds. Then, $(2.9)$ reduces to

$$
E_{k+1}=E_{k}\left(I-\frac{q_{k} q_{k}^{*}}{q_{k}^{*} q_{k}} A\right) .
$$

Remark that Broyden's bad update is well defined as long as $\Delta_{k} \neq 0$. In particular, no additional restrictions for $H_{0}$ are needed.

For the special error matrix

$$
\widehat{E}_{k}:=A E_{k} A^{-1}=I-A H_{k},
$$

(2.21) leads to the update formula

$$
\widehat{E}_{k+1}=\widehat{E}_{k}\left(I-\frac{q_{k} q_{k}^{*}}{q_{k}^{*} q_{k}}\right) .
$$

From (2.23), it follows that $H_{k+1}$ is an improved preconditioner, in the sense that

$$
\left\|\widehat{E}_{k+1}\right\| \leq\left\|\widehat{E}_{k}\right\|
$$

and

$$
\left\|\widehat{E}_{k+1}\right\|_{F}^{2}=\left\|\widehat{E}_{k}\right\|_{F}^{2}-\frac{\left\|\widehat{E}_{k} q_{k}\right\|^{2}}{\left\|q_{k}\right\|^{2}}
$$


(C) A third obvious choice for $v_{k}$ in (2.2) is

$$
v_{k}:=z_{k} .
$$

The corresponding update (2.9) for the error matrix is

$$
E_{k+1}=E_{k}\left(I-\frac{\Delta_{k} z_{k}^{*}}{z_{k}^{*} z_{k}} H_{k} A\right) .
$$

Here, one needs to ensure $z_{k} \neq 0$. Obviously, this is guaranteed if $H_{k}$ is nonsingular and $x_{k} \neq x$. If $H_{k}$ is nonsingular, then (2.26) can be rewritten in terms of an orthogonal projection as follows:

$$
E_{k+1}=E_{k}\left(H_{k} A\right)^{-1}\left(I-\frac{z_{k} z_{k}^{*}}{z_{k}^{*} z_{k}}\right) H_{k} A .
$$

However, unlike as for updates (A) and (B), (2.27) does not imply a reduction property of some "natural" measure for the preconditioner $H_{k}$. This suggests that this type of update is not competitive with Broyden's good and bad ones. Indeed, this was confirmed by our numerical experiments.

\subsection{Line Search Principles}

In this section, the selection of the step length $t_{k}$ in part c) of Algorithm 2.1 is discussed. Ideally, one would like to choose $t_{k}$ such that

$$
\left\|e_{k+1}\left(t_{k}\right)\right\|=\min _{t \in \mathbb{C}}\left\|e_{k+1}(t)\right\|
$$

where

$$
e_{k+1}(t):=e_{k}-t \Delta_{k} .
$$

Unfortunately, since $x$ and hence $e_{k}$ is unavailable, the step length defined by (2.28) can not be computed. However, in view of

$$
e_{k+1}(t)=A^{-1} r_{k+1}(t) \text { where } r_{k+1}(t):=r_{k}-t q_{k},
$$

(2.28) can be satisfied at least approximately by choosing $t_{k}$ such that

$$
\left\|C_{k} r_{k+1}\left(t_{k}\right)\right\|=\min _{t \in \mathbb{C}}\left\|C_{k} r_{k+1}(t)\right\| .
$$

Here $C_{k}$ is some approximate inverse of $A$. At iteration $k$ of Algorithm 2.1, there are three natural choices for $C_{k}$, namely $H_{k+1}, H_{k}$, or simply $C_{k}=I$, which lead to the line search principles (a), (c), or (b), respectively. Next, these three strategies are discussed. 
(a) With $C_{k}=H_{k+1}$ and $\Delta_{k+1}=H_{k+1} r_{k+1},(2.30)$ reads as follows:

$$
\left\|\Delta_{k+1}\left(t_{k}\right)\right\|=\min _{t \in \mathbb{C}}\left\|\Delta_{k+1}(t)\right\|
$$

Using (2.6b) and the second relation in (2.29), one readily verifies that (2.31) is equivalent to

$$
\Delta_{k}^{*} \Delta_{k+1}=0 \text { where } \Delta_{k+1}=\left(1+\tau_{k}\right) \Delta_{k}-\tau_{k} z_{k}-t_{k} \Delta_{k} .
$$

Recall that $\tau_{k}$ was defined in (2.5) and note that $\tau_{k}$ still depends on the particular choice of the rank-1 update (2.2).

Finally, from (2.32), it follows that the step length for the line search principle (2.31) is given by

$$
t_{k}=\tilde{t}_{k}:=1+\tau_{k}-\tau_{k} \frac{\Delta_{k}^{*} z_{k}}{\Delta_{k}^{*} \Delta_{k}} .
$$

Note that for the special case, $v_{k}=\Delta_{k}$, of Broyden's good update, (2.33) leads to

$$
\tilde{t}_{k}=\tau_{k}=\frac{\Delta_{k}^{*} \Delta_{k}}{\Delta_{k}^{*} z_{k}}
$$

(b) For $C_{k}=I,(2.30)$ reduces to

$$
\left\|r_{k+1}\left(t_{k}\right)\right\|=\min _{t \in \mathbb{C}}\left\|r_{k+1}(t)\right\|
$$

or, equivalently,

$$
q_{k}^{*} r_{k+1}=0 \text { where } r_{k+1}=r_{k}-t_{k} q_{k} .
$$

Hence, by (2.36), the minimization principle (2.35) leads to

$$
t_{k}=\widehat{t}_{k}:=\frac{q_{k}^{*} r_{k}}{q_{k}^{*} q_{k}} .
$$

Remark that for Broyden's bad update, (B), one has

$$
\widehat{t}_{k}=\tau_{k} \text {. }
$$

(c) With $C_{k}=H_{k},(2.30)$ specifies to

$$
\left\|H_{k} r_{k+1}\left(t_{k}\right)\right\|=\min _{t \in \mathbb{C}}\left\|H_{k} r_{k+1}(t)\right\|
$$


By rewriting (2.39) in the form

$$
z_{k}^{*} H_{k} r_{k+1}=0 \text { where } H_{k} r_{k+1}=\Delta_{k}-t_{k} z_{k},
$$

it follows that

$$
t_{k}=t_{k}^{\circ}:=\frac{z_{k}^{*} \Delta_{k}}{z_{k}^{*} z_{k}}
$$

Here, for update $(\mathrm{C})$, one has

$$
t_{k}^{\circ}=\tau_{k}
$$

Notice that, in view of (2.34), (2.38), and (2.41), the choice $t_{k}=\tau_{k}$ for the step length leads to a natural coupling of the three special rank-1 updates (A), (B), and (C) with the line search principles (a), (b), and (c), respectively.

More general, for $t_{k}=\tau_{k}$, the following properties hold.

Lemma 2.4 In Algorithm 2.1, let $t_{k}=\tau_{k}$ be chosen and assume that $v_{k}^{*} z_{k} \neq 0$. Then:

a) The iterate $x_{k+1}$ is uniquely defined by the Galerkin type condition

$$
H_{k} r_{k+1} \perp v_{k} \text { and } x_{k+1} \in x_{k}+\operatorname{span}\left\{\Delta_{k}\right\}
$$

b) $H_{k+1} r_{k+1}=H_{k} r_{k+1}$.

Proof. By the second condition in (2.42), $x_{k+1}=x_{k}+t \Delta_{k}$ and thus

$$
H_{k} r_{k+1}=\Delta_{k}-t z_{k}
$$

for some $t \in \mathbb{C}$. Together with the definition of $\tau_{k}$ in (2.5), it follows that

$$
v_{k}^{*} H_{k} r_{k+1}=v_{k}^{*} \Delta_{k}-t v_{k}^{*} z_{k}=0 \Leftrightarrow t=\frac{v_{k}^{*} \Delta_{k}}{v_{k}^{*} z_{k}}=\tau_{k},
$$

and this concludes the proof of a).

Next, one easily verifies that

$$
H_{k} r_{k+1}=\left(1-t_{k}\right) \Delta_{k}+t_{k} E_{k} \Delta_{k} .
$$

By comparing (2.6b) and (2.43), one obtains the relation stated in b).

Remark that the classical step length used in combination with Broyden's update $(2.2)$ is $t_{k} \equiv 1$. Somewhat surprisingly, this choice guarantees that the resulting method - at least in theory - terminates after at most $2 n$ steps with the exact solution of (1.1), as was shown by GAY [9] (cf. also [10]). Obviously, this finite termination property is not of practical importance for large sparse linear systems. Here, we take another look at the choice $t_{k} \equiv 1$. 
Lemma 2.5 In Algorithm 2.1, assume that $v_{k}^{*} z_{k} \neq 0$. Let $x_{k}$ and $x_{k+2}$ be the iterates generated by two successive steps of Algorithm 2.1 with step length $t_{k}=t_{k+1}=1$. Then:

$$
x_{k+2}=\widehat{x}_{k+1}+H_{k} \widehat{r}_{k+1}, \quad r_{k+2}=\left(I-A H_{k}\right) \widehat{r}_{k+1},
$$

where

$$
\widehat{x}_{k+1}=x_{k}+\tau_{k} \Delta_{k}, \quad \widehat{r}_{k+1}=\left(I-\tau_{k} A H_{k}\right) r_{k}
$$

and

$$
\tau_{k}=\frac{v_{k}^{*} \Delta_{k}}{v_{k}^{*} z_{k}}=\frac{\left(H_{k}^{*} v_{k}\right)^{*} r_{k}}{\left(H_{k}^{*} v_{k}\right)^{*} q_{k}}
$$

Proof. Since $t_{k}=t_{k+1}=1$, we have

$$
x_{k+2}=x_{k}+\Delta_{k}+\Delta_{k+1} .
$$

For $t_{k}=1$, the first identity in $(2.6 \mathrm{~b})$ reduces to $\Delta_{k+1}=\tau_{k}\left(I-H_{k} A\right) \Delta_{k}$ and, thus, (2.46) can be rewritten as

$$
x_{k+2}=x_{k}+\tau_{k} \Delta_{k}+H_{k}\left(I-\tau_{k} A H_{k}\right) r_{k} .
$$

Now, (2.44) and (2.45) readily follow from (2.47).

Note that, in view of part a) of Lemma 2.4 , the intermediate quantity $\hat{x}_{k+1}$, $(2.45)$, is just the Galerkin iterate in the sense of (2.42).

Therefore, Lemma 2.5 shows that, by combining two successive steps, Algorithm 2.1 with $t_{k} \equiv 1$ can be interpreted as follows. At the beginning of step $k$, the approximate solution $x_{k}$ and the preconditioner $H_{k}$ are available. From these quantities, the iterate $x_{k+2}$ of step $k+2$ is obtained by applying one Galerkin step, namely (2.45), followed by one step of Richardson iteration, namely (2.44), to the preconditioned linear system

$$
H_{k} A x=H_{k} b .
$$

In general, the "virtual" iterate $\widehat{x}_{k+1}$ and the actual iterate $x_{k+1}$ are different.

Note that (2.44) is a Richardson step without line search. In particular, if $H_{k}$ - as is to be expected in the early stage of the iteration - is not yet a good approximation to $A^{-1}$, then (2.44) will lead to an increase rather than a decrease of $\left\|r_{k+2}\right\|$. In order to prevent such undesirable effects, it appears preferable to combine Broyden's update with the line search principles (a), (b), or (c), instead of using $t_{k} \equiv 1$. 


\section{Convergence Analysis}

In principle, Algorithm 2.1 could be implemented with any of the 9 combinations $(\mathrm{Aa}), \ldots,(\mathrm{Cc})$ of rank-1 updates (A), (B), and (C) with line search strategies (a), (b), and (c). As already mentioned in Section 2.2, the update (C) is not competitive with (A) and (B), and, therefore, (C) is dropped here. Among the remaining 6 combinations, only the pairs $(\mathrm{Aa}),(\mathrm{Bb})$, and $(\mathrm{Ac})$ will be considered.

As a first step, the following auxiliary result for the case of the line search principle (b) is established.

Lemma 3.1 In the general Algorithm 2.1, let the step length (2.37), $t_{k}=\widehat{t}_{k}$, be chosen. Then,

$$
\frac{\left\|r_{k+1}\right\|}{\left\|r_{k}\right\|} \leq \frac{\left\|\widehat{E}_{k} r_{k}\right\|}{\left\|r_{k}\right\|} \leq\left\|\widehat{E}_{k}\right\|
$$

with $\widehat{E}_{k}=I-A H_{k}$ defined as in (2.22).

Proof. From part c) of Algorithm 2.1 and (2.37), one obtains

$$
r_{k+1}=r_{k}-\widehat{t}_{k} q_{k}=r_{k}-\frac{q_{k}^{*} r_{k}}{q_{k}^{*} q_{k}} q_{k}=\left(I-\frac{q_{k} q_{k}^{*}}{q_{k}^{*} q_{k}}\right) r_{k}=\left(I-\frac{q_{k} q_{k}^{*}}{q_{k}^{*} q_{k}}\right)\left(r_{k}-q_{k}\right) \text {. }
$$

Since

$$
r_{k}-q_{k}=\widehat{E}_{k} r_{k}
$$

it follows that

$$
\left\|r_{k+1}\right\|=\left\|\left(I-\frac{q_{k} q_{k}^{*}}{q_{k}^{*} q_{k}}\right) \widehat{E}_{k} r_{k}\right\| \leq\left\|\widehat{E}_{k} r_{k}\right\| \leq\left\|\widehat{E}_{k}\right\| \cdot\left\|r_{k}\right\|,
$$

and thus (3.1) holds.

Recall from Section 2.2 that the error matrix $\widehat{E}_{k}$ is closely connected with Broyden's bad update (B), cf. (2.23)-(2.25). In the following section, Lemma 3.1 will be used to obtain a convergence result for update $(B)$.

\subsection{Broyden's Bad Update}

Theorem 3.2 (B-update)

Consider Algorithm 2.1 with update (B) and step length $t_{k}=\widehat{t}_{k}=\tau_{k},(2.37)$, or $t_{k}=1$. Assume that

$$
\left\|\widehat{E}_{0}\right\| \leq \widehat{\delta}_{0}<1 \text {. }
$$


Then, the iteration converges globally satisfying

$$
\frac{\left\|A e_{k+1}\right\|}{\left\|A e_{k}\right\|}=\frac{\left\|r_{k+1}\right\|}{\left\|r_{k}\right\|} \leq \frac{\left\|\widehat{E}_{k} r_{k}\right\|}{\left\|r_{k}\right\|} \leq \widehat{\delta}_{0}<1
$$

and

$$
\tau_{k}>0 \text { for } r_{k} \neq 0 .
$$

Moreover, if $r_{k} \neq 0$ for all $k=0,1, \ldots$, then,

$$
\lim _{k \rightarrow \infty} t_{k}=1
$$

and the convergence is superlinear in the sense that

$$
\lim _{k \rightarrow \infty} \frac{\left\|A e_{k+1}\right\|}{\left\|A e_{k}\right\|}=0
$$

Proof. First, global convergence is shown for $t_{k}=\tau_{k}$. By Lemma 3.1,

$$
\frac{\left\|r_{k+1}\right\|}{\left\|r_{k}\right\|} \leq \frac{\left\|\widehat{E}_{k} r_{k}\right\|}{\left\|r_{k}\right\|} \leq\left\|\widehat{E}_{k}\right\| \text {. }
$$

In view of (2.24), (3.7) implies

$$
\frac{\left\|r_{k+1}\right\|}{\left\|r_{k}\right\|} \leq\left\|\widehat{E}_{k}\right\| \leq\left\|\widehat{E}_{0}\right\| \leq \widehat{\delta}_{0}<1 .
$$

By combining (3.7) and (3.8), the statement in (3.3) follows. By (2.37), (2.38), and (3.2), the step length $\hat{t}_{k}=\tau_{k}$ satisfies

$$
\tau_{k}=\frac{q_{k}^{*} r_{k}}{q_{k}^{*} q_{k}}=\frac{\left\|r_{k}\right\|^{2}}{\left\|q_{k}\right\|^{2}}-\frac{r_{k}^{*} \widehat{E}_{k}^{*} r_{k}}{\left\|q_{k}\right\|^{2}} .
$$

Using (3.7), one deduces from (3.9) that

$$
\tau_{k} \geq \frac{\left\|r_{k}\right\|^{2}}{\left\|q_{k}\right\|^{2}}-\frac{\left|r_{k}^{*} \widehat{E}_{k} r_{k}\right|}{\left\|q_{k}\right\|^{2}} \geq\left(1-\left\|\widehat{E}_{k}\right\|\right) \frac{\left\|r_{k}\right\|^{2}}{\left\|q_{k}\right\|^{2}}>0 \text { for } r_{k} \neq 0,
$$

and (3.4) holds true.

Next, based on the Frobenius norm property (2.25), superlinear convergence is shown. Following the proof technique of Broyden/DENnIS/Moré [2], one obtains

$$
\lim _{k \rightarrow \infty} \frac{\left\|\widehat{E}_{k} q_{k}\right\|}{\left\|q_{k}\right\|}=0
$$


By (3.8), the assumption $\widehat{\delta}_{0}<1$ guarantees that the matrix $\left(I-\widehat{E}_{k}\right)$ is nonsingular. Thus the relation (3.2) can be rewritten as

$$
r_{k}=\left(I-\widehat{E}_{k}\right)^{-1} q_{k} .
$$

Using (3.11) and (3.8), one readily verifies that

$$
\frac{\left\|\widehat{E}_{k} r_{k}\right\|}{\left\|r_{k}\right\|}=\frac{\left\|\widehat{E}_{k}\left(I-\widehat{E}_{k}\right)^{-1} q_{k}\right\|}{\left\|\left(I-\widehat{E}_{k}\right)^{-1} q_{k}\right\|} \leq \frac{1+\left\|\widehat{E}_{k}\right\|}{1-\left\|\widehat{E}_{k}\right\|} \cdot \frac{\left\|\widehat{E}_{k} q_{k}\right\|}{\left\|q_{k}\right\|} \leq \frac{1+\widehat{\delta}_{0}}{1-\widehat{\delta}_{0}} \cdot \frac{\left\|\widehat{E}_{k} q_{k}\right\|}{\left\|q_{k}\right\|}
$$

Therefore, by means of (3.7) and (3.10), one concludes that

$$
\lim _{k \rightarrow \infty} \frac{\left\|r_{k+1}\right\|}{\left\|r_{k}\right\|} \leq \frac{1+\widehat{\delta}_{0}}{1-\widehat{\delta}_{0}} \lim _{k \rightarrow \infty} \frac{\left\|\widehat{E}_{k} q_{k}\right\|}{\left\|q_{k}\right\|}=0
$$

which immediately yields (3.6). Similarly, from

$$
t_{k}=\frac{q_{k}^{*} r_{k}}{q_{k}^{*} q_{k}}=\frac{q_{k}^{*}\left(I-\widehat{E}_{k}\right)^{-1} q_{k}}{q_{k}^{*} q_{k}}
$$

one deduces that

$$
\left|t_{k}-1\right| \leq \frac{1}{1-\widehat{\delta}_{0}} \cdot \frac{\left\|\widehat{E}_{k} q_{k}\right\|}{\left\|q_{k}\right\|}
$$

Therefore, (3.10) implies

$$
\lim _{k \rightarrow \infty}\left|t_{k}-1\right|=0
$$

which confirms (3.5).

For the case $t_{k}=1$, the relation $(2.6 \mathrm{a})$ reduces to

$$
e_{k+1}=E_{k} e_{k}
$$

which is equivalent to

$$
r_{k+1}=\widehat{E}_{k} r_{k} .
$$

Now (3.12), also yields (3.7). The rest of the proof can just be copied.

\subsection{Broyden's Good Update}

Theorem 3.3 (A-update)

Consider Algorithm 2.1 with update (A) and line search either (a) or (c). Assume that

$$
\left\|\tilde{E}_{0}\right\| \leq \tilde{\delta}_{0}<\frac{1}{3}
$$


Then, the iteration converges globally satisfying

$$
\frac{\left\|e_{k+1}\right\|}{\left\|e_{k}\right\|} \leq \frac{\frac{\left\|\tilde{E}_{k} z_{k}\right\|}{\left\|z_{k}\right\|}+\frac{\left\|\widetilde{E}_{k} \Delta_{k}\right\|}{\left\|\Delta_{k}\right\|}}{1-\frac{\left\|\widetilde{E}_{k} \Delta_{k}\right\|}{\left\|\Delta_{k}\right\|}} \leq \frac{2 \tilde{\delta}_{0}}{1-\widetilde{\delta}_{0}}<1
$$

and

$$
\tau_{k}>0 \text { for } e_{k} \neq 0 \text {. }
$$

Moreover, if $e_{k} \neq 0$ for all $k=0,1, \ldots$, then the convergence is superlinear with

$$
\lim _{k \rightarrow \infty} \frac{\left\|e_{k+1}\right\|}{\left\|e_{k}\right\|}=0
$$

and

$$
\lim _{k \rightarrow \infty} t_{k}=1
$$

Proof. First, line search (a) with $t_{k}=\tau_{k}$ (cf. (2.34)) is considered. Rewriting (2.6a) in terms of $\widetilde{E}_{k}$ yields

$$
e_{k+1}=\left(1-\tau_{k}\right) \Delta_{k}-\widetilde{E}_{k} \Delta_{k}
$$

By means of the relations

$$
1-\tau_{k}=1-\frac{\Delta_{k}^{*} \Delta_{k}}{\Delta_{k}^{*} z_{k}}=\frac{\Delta_{k}^{*}\left(z_{k}-\Delta_{k}\right)}{\Delta_{k}^{*} z_{k}}
$$

and

$$
\Delta_{k}=\left(I-\tilde{E}_{k}\right) z_{k}
$$

one obtains from (3.18)

$$
e_{k+1}=\frac{\Delta_{k}^{*} \tilde{E}_{k} z_{k}}{\Delta_{k}^{*} z_{k}} \cdot \Delta_{k}-\widetilde{E}_{k} \Delta_{k}
$$

Moreover, using the formula (3.19) once more, one easily verifies that

$$
\begin{aligned}
& \frac{\left|\Delta_{k}^{*} \widetilde{E}_{k} z_{k}\right|}{\left|\Delta_{k}^{*} z_{k}\right|}=\frac{\left|\left(z_{k}-\widetilde{E}_{k} z_{k}\right)^{*} \widetilde{E}_{k} z_{k}\right|}{\left|\left(z_{k}-\widetilde{E}_{k} z_{k}\right)^{*} z_{k}\right|} \\
& \quad=\frac{\left|z_{k}^{*} \widetilde{E}_{k} z_{k}\right|}{\left\|z_{k}\right\|^{2}} \cdot \frac{\left|1-\frac{\left\|\widetilde{E}_{k} z_{k}\right\|^{2}}{z_{k}^{*} \widetilde{E}_{k} z_{k}}\right|}{\left|1-\frac{z_{k}^{*} \widetilde{E}_{k} z_{k}}{\left\|z_{k}\right\|^{2}}\right|} \leq \widetilde{\varepsilon}_{k} \cdot \frac{1-\widetilde{\varepsilon}_{k}}{1-\widetilde{\varepsilon}_{k}}=\widetilde{\varepsilon}_{k},
\end{aligned}
$$


where

$$
\tilde{\varepsilon}_{k}:=\frac{\left\|\tilde{E}_{k} z_{k}\right\|}{\left\|z_{k}\right\|} \leq\left\|\tilde{E}_{k}\right\| \leq \tilde{\delta}_{0} .
$$

With these inequalities, (3.20) leads to the estimates

$$
\frac{\left\|e_{k+1}\right\|}{\left\|\Delta_{k}\right\|} \leq \widetilde{\varepsilon}_{k}+\frac{\left\|\widetilde{E}_{k} \Delta_{k}\right\|}{\left\|\Delta_{k}\right\|} \leq 2 \widetilde{\delta}_{0} .
$$

Finally, with

$$
e_{k}=\left(I+\widetilde{E}_{k}\right) \Delta_{k}
$$

one obtains

$$
\left\|e_{k}\right\| \geq\left(1-\frac{\left\|\tilde{E}_{k} \Delta_{k}\right\|}{\left\|\Delta_{k}\right\|}\right)\left\|\Delta_{k}\right\| \geq\left(1-\tilde{\delta}_{0}\right)\left\|\Delta_{k}\right\| .
$$

By combining (3.21) and (3.22), one ends up with (3.14).

Similarly, one shows

$$
\tau_{k}=1-\frac{\Delta_{k}^{*} \widetilde{E}_{k} z_{k}}{\Delta_{k}^{*} z_{k}} \geq 1-\widetilde{\varepsilon}_{k}
$$

which certainly confirms the assertion (3.15).

In order to prove superlinear convergence, first remark that the Frobenius norm result (2.14) implies

$$
\lim _{k \rightarrow \infty} \frac{\left\|\widetilde{E}_{k} \Delta_{k}\right\|}{\left\|\Delta_{k}\right\|}=0
$$

Along lines similar as in the proof of Theorem 3.2 , one then verifies that

$$
\lim _{k \rightarrow \infty} \widetilde{\varepsilon}_{k}=0
$$

Now, by using (3.23), (3.24), and the estimates in (3.14) of this theorem, one obtains (3.16) and, with

$$
\left|t_{k}-1\right| \leq \widetilde{\varepsilon}_{k}
$$

also (3.17). This completes the proof for line search (a).

Next, consider the line search principle (c) where, by (2.40),

$$
t_{k}=t_{k}^{\circ}=\frac{z_{k}^{*} \Delta_{k}}{z_{k}^{*} z_{k}}
$$

As before, one starts with

$$
e_{k+1}=\left(1-t_{k}\right) \Delta_{k}-\tilde{E}_{k} \Delta_{k}
$$


which now leads to

$$
e_{k+1}=\frac{z_{k}^{*} \tilde{E}_{k}^{*} z_{k}}{z_{k}^{*} z_{k}} \Delta_{k}-\widetilde{E}_{k} \Delta_{k}
$$

From this, one derives the estimate

$$
\frac{\left\|e_{k+1}\right\|}{\left\|\Delta_{k}\right\|} \leq \widetilde{\varepsilon}_{k}+\frac{\left\|\tilde{E}_{k} \Delta_{k}\right\|}{\left\|\Delta_{k}\right\|},
$$

which is the same as (3.21). The rest of the proof can essentially be copied.

For the choice $t_{k} \equiv 1$, Broyden's classical good method is obtained. The convergence behavior of this algorithm is studied in BROYDEN/DENNIS/Moré [2]. In this case, the assumption (3.13) can be relaxed to $\widetilde{\delta}_{0}<\frac{1}{2}$. As already mentioned, the choice $t_{k} \equiv 1$ guarantees that Broyden's good method stops after at most $2 n$ steps. A slight modification, the so-called projected Broyden's method, even terminates after at most $n$ iterations. This algorithm is analyzed in GAY/SCHNABEL [8].

Conjecture. The authors were unable to get rid of the factor 2 in (3.14). If this factor drops, then only $\widetilde{\delta}_{0}<\frac{1}{2}$ would be required - which seems to be more reasonable in view of $(2.20)$.

\subsection{An Illustrative Example}

In this section, we discuss a simple illustrative example, namely a convectiondiffusion problem in 1-D. Consider the ODE boundary value problem

$$
\begin{aligned}
& \text { a) }-u^{\prime \prime}+\beta u^{\prime}=0 \text { on }(0,1), \\
& \text { b) } u(0)=1, u(1)=0 .
\end{aligned}
$$

By using upwind discretization on a uniform grid with step size $h=1 / n,(3.25)$ leads to a linear system $A x=b$ with the diagonally dominant tridiagonal matrix

$$
A:=\left[\begin{array}{cccc}
2+\beta h & -1 & & \\
-(1+\beta h) & \ddots & \ddots & \\
& \ddots & \ddots & -1 \\
& & -(1+\beta h) & 2+\beta h
\end{array}\right] .
$$

Let $n=50$ and set $b=(1,0, \ldots, 0)^{T}$. Moreover, choose $x_{0}$ as the prolongation obtained from the exact solution on the coarser grid $h=1 / 25$. For $H_{0}$, we 
chose simple diagonal preconditioning as in (5.1). In this case, one is able to compute all quantities of interest directly and to compare the convergence theory of Sections 3.1 and 3.2 with the actual behavior of the algorithms - see Table 3.1.

\begin{tabular}{|l|r|l|c|c|c|}
\hline & $\left\|\tilde{E}_{0}\right\|$ & $\left\|\widehat{E}_{0}\right\|$ & $\left\|\widehat{E}_{0} r_{0}\right\| /\left\|r_{0}\right\|$ & $\left\|\widetilde{E}_{0} z_{0}\right\| /\left\|z_{0}\right\|$ & $\left\|\widetilde{E}_{0} \Delta_{0}\right\| /\left\|\Delta_{0}\right\|$ \\
\hline$\beta=5$ & 415 & 0.99 & 0.53 & 0.43 & 2.72 \\
\hline$\beta=100$ & 64 & 0.99 & 0.37 & 0.28 & 0.24 \\
\hline
\end{tabular}

Table 3.1: Quantities used in convergence theory of Sections 3.1 and 3.2 for Example (3.25).

These results seem to justify the relaxation of the rather restrictive convergence criteria in Sections 3.1 and 3.2 - compare (2.16) and (2.17) in the light of (2.18), (2.20), and (3.13).

In Fig. 3.1 and Fig. 3.2, the convergence history of 3 codes (see Section 5 for a description of these codes) is compared - both in terms of the residual norms $\left\|r_{k}\right\|$ and the error norms $\left\|e_{k}\right\|$.

In this example, both GMRES and the "bad Broyden" code BB successively reduce the residual norm, whereas the "good Broyden" code GB reduces the error norm - a property that has been shown to hold at least asymptotically without a storage restriction: just compare the minimization property (2.31), $\left\|\Delta_{k+1}\right\|=\min$, for $t_{k}=\tau_{k}$ with the asymptotic property (see (4.2) below) $\left\|\Delta_{k+1}\right\| \doteq\left\|e_{k+1}\right\|$ for $\tau_{k} \doteq 1$. As an illustration, Fig. 3.3 gives a comparison of the true and estimated errors. 


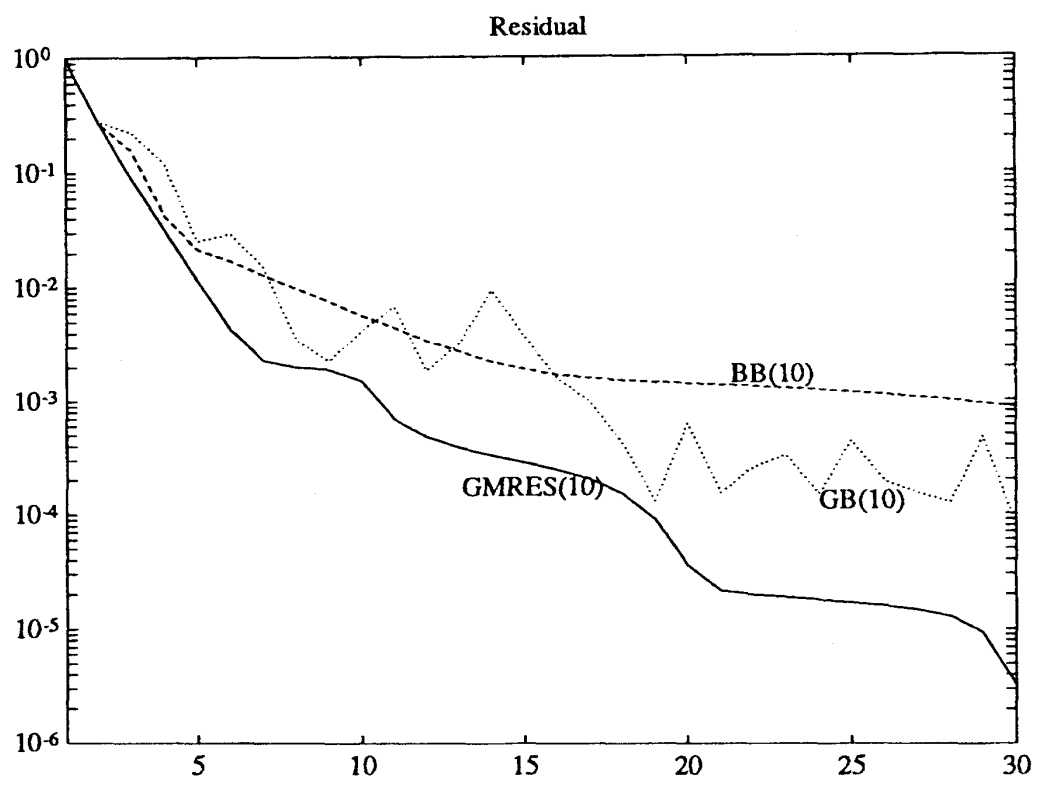

Figure 3.1: Comparative residual norms $\left\|r_{k}\right\|_{2}$ for 3 iterative solvers for Example (3.25).

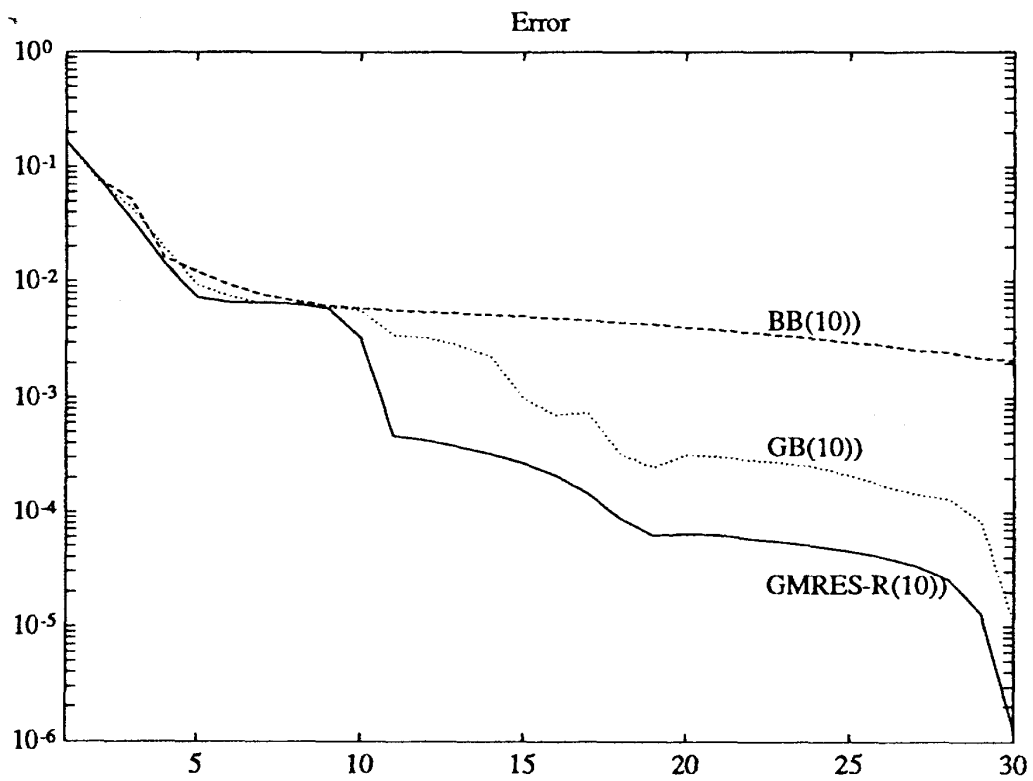

Figure 3.2: Comparative error norms $\left\|e_{k}\right\|_{2}$ for 3 iterative solvers with $k_{\max }=10$ for Example (3.25). 


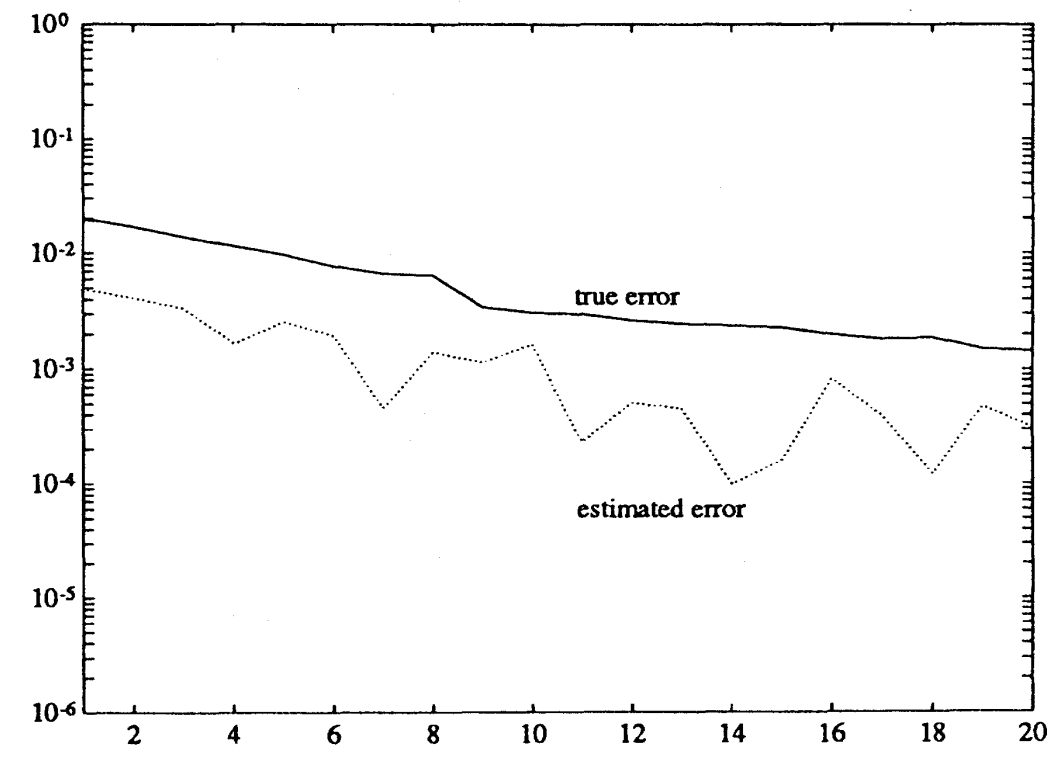

Figure 3.3: Iterative behavior of true errors $\left\|e_{k}\right\|$ and estimated errors $\left\|\Delta_{k}\right\|$ for $\mathrm{GB}(3)$ in Example (3.25). 


\section{Details of Realization}

The secant methods based on Algorithm 2.1 with update either (A) or (B) are, of course, implemented in a storage saving compact form.

\section{Algorithm (A): "Good Broyden"}

$$
\begin{aligned}
& \text { Start: a) } r_{0}:=b-A x_{0} \\
& \Delta_{0}:=H_{0} r_{0} \\
& \sigma_{0}:=\Delta_{0}^{*} \Delta_{0} \\
& \tilde{z}_{0}:=H_{0} q_{k} \\
& \gamma_{k}:=\Delta_{k}^{*} z_{k} \\
& \tau_{k}:=\sigma_{k} / \gamma_{k} \\
& t_{k}:=\tau_{k} \text { or } t_{k}:=\widehat{t}_{k}=q_{k}^{*} r_{k} / q_{k}^{*} q_{k} \text { or } t_{k}:=1 \\
& x_{k+1}:=x_{k}+t_{k} \Delta_{k} \\
& r_{k+1}:=r_{k}-t_{k} q_{k} \\
& \Delta_{k+1}:=\left(1-t_{k}+\tau_{k}\right) \Delta_{k}-\tau_{k} z_{k} \\
& \sigma_{k+1}:=\Delta_{k+1}^{*} \Delta_{k+1}
\end{aligned}
$$

Array storage. The above implementation requires to store (up to iteration step $k$ ) the vectors

$$
\Delta_{0}, \ldots, \Delta_{k}, q, z=\dot{z},
$$

for step length $t_{k}=\tau_{k}$ or $t_{k}=1$ and, in addition, $r=r_{k}$ in the case of step length $t_{k}=\widetilde{t}_{k}$, which sum up to

$$
(k+2) n \text { or }(k+3) n
$$

storage places. 
Operation count. Per iteration step $k$ one needs 1 matrix-vector multiplication, 1 solution of a preconditioned system of the form $B_{0} z=q$ in order to obtain $\widetilde{z}_{0}$ in $\left.\mathrm{b}\right)$, and $(2 k+7) n$ multiplications. Obviously, the inner loop vectorizes.

Termination criteria. Under the assumptions of Theorem 3.3 (cf. (3.22)), one has

$$
\left(1-\frac{\left\|\tilde{E}_{k} \Delta_{k}\right\|}{\left\|\Delta_{k}\right\|}\right)\left\|\Delta_{k}\right\| \leq\left\|e_{k}\right\| \leq\left(1+\frac{\left\|\widetilde{E}_{k} \Delta_{k}\right\|}{\left\|\Delta_{k}\right\|}\right)\left\|\Delta_{k}\right\|,
$$

which means that, at least asymptotically, $\left\|\Delta_{k}\right\|$ is a reasonable computationally available estimate for $\left\|e_{k}\right\|$ to be written as

$$
\left\|\Delta_{k}\right\| \doteq\left\|e_{k}\right\|
$$

This motivates the convergence criterion

$$
\frac{\sqrt{\sigma_{k+1}}}{\left\|x_{k+1}\right\|} \leq \varepsilon
$$

where $\varepsilon$ is some relative accuracy parameter to be specified by the user.

In order to ensure that $H_{k+1} A$ is nonsingular, recall condition (2.17), which reads $\varepsilon_{k}<1$ in the notation of Section 2.2. By replacing this condition by the stricter one

$$
\varepsilon_{k} \leq 1-\frac{1}{\tau_{\max }}
$$

we arrive at a restart condition

$$
\tau_{k} \leq 0 \quad \text { or } \quad \tau_{k}>\tau_{\max }
$$

which can be easily monitored. Here, $\tau_{\max }$ is some internal parameter, and we have chosen $\tau_{\max }=10$ in all the numerical experiments described in this paper.

Remark 1. The convergence criterion (4.3) nicely agrees with requirements needed in the global inexact Newton algorithm for nonlinear problems as given by Deuflhard [4].

Remark 2. Clearly, the Euclidean inner product in Algorithm (A) can be replaced by any other inner product $(\cdot, \cdot)$ - possibly scaled and certainly depending on the problem to be solved. 


\section{Algorithm (B): "Bad Broyden"}

$$
\begin{aligned}
& \text { Start: a) } r_{0}:=b-A x_{0} \\
& \Delta_{0}:=H_{0} r_{0} \\
& \text { Iteration loop: } k=0,1, \ldots \text { : } \\
& \text { b) } q_{k}:=A \Delta_{k} \\
& \tilde{z}_{0}:=H_{0} q_{k} \\
& \text { Update loop: } i=0, \ldots, k-1 \text { (for } k \geq 1 \text { ) } \\
& \text { c) } \tilde{z}_{i+1}:=\tilde{z}_{i}+\frac{q_{i}^{*} q_{k}}{\beta_{i} t_{i}}\left(\Delta_{i+1}-\left(1-t_{i}\right) \Delta_{i}\right) \\
& \text { d) } z_{k}:=\tilde{z}_{k} \\
& \beta_{k}:=q_{k}^{*} q_{k} \\
& t_{k}:=\frac{r_{k}^{*} q_{k}}{\beta_{k}} \\
& x_{k+1}:=x_{k}+t_{k} \Delta_{k} \\
& r_{k+1}:=r_{k}-t_{k} q_{k} \\
& \Delta_{k+1}:=\Delta_{k}-t_{k} z_{k}
\end{aligned}
$$

The version for $t_{k}=1$ was ignored for obvious reasons.

Array Storage. The implementation of this algorithm requires to store (up to iteration step $k$ ) the vectors

$$
\Delta_{0}, \ldots, \Delta_{k}, q_{0}, \ldots, q_{k}, z=\widetilde{z}, r
$$

which sums up to

$$
(2 k+2) n
$$

storage places - to be compared with (4.1).

Operation count. Per iterative step $k$ one needs 1 matrix-vector multiplication, again solution of 1 linear preconditioned system with $B_{0}$ as coefficient matrix, and $(2 k+8) n$ multiplications. Once more, the inner loop easily vectorizes. 
Termination criteria. Since update (B) is closely connected with minimization principle (2.35), the convergence criterion for Algorithm (B) will be based on the residual norm. In view of the property (for $t_{k}=\tau_{k}$ )

$$
r_{k+1}^{*} r_{k+1}=r_{k}^{*} r_{k}-t_{k}^{2} q_{k}^{*} q_{k},
$$

Algorithm (B) is stopped as soon as

$$
\left\|r_{k+1}\right\| \leq \varepsilon\left\|r_{0}\right\|
$$

is reached. Again, $\varepsilon$ is to be specified by the user. Moreover, the iteration is restarted, if

$$
\left|t_{k}\right| \cdot\left\|q_{k}\right\|<\varepsilon\left\|r_{0}\right\|
$$

Note that in view of Theorem 3.2 , the iteration would need to be just terminated, if

$$
\frac{\left\|\widehat{E}_{k} r_{k}\right\|}{\left\|r_{k}\right\|}=\frac{\left\|q_{k}-r_{k}\right\|}{\left\|r_{k}\right\|}>1,
$$

which can be shown to be equivalent to the condition

$$
t_{k}=\tau_{k}<\frac{1}{2} .
$$

\section{Restricted storage versions}

For large $n$, one needs to restrict storage to some $m \cdot n$ such that

$$
\begin{array}{ll}
k_{\max }+2=m & \text { for }(A a), \\
2 k_{\max }+2=m & \text { for }(B b) .
\end{array}
$$

Several options are possible to satisfy this restriction.

(I) Both Algorithms (A) and (B) can be just restarted after $k_{\max }$ iterations using $x_{k_{\max }}$ as the new starting guess $x_{0}$. Under the assumptions of the convergence theorems in Sections 3.1 and 3.2, these restricted variants can be shown to converge linearly.

(II) Both (A) and (B) can be modified by restricting the update loop to indices

$$
i=0, \ldots, k_{\max }-1 \text {.(initial window). }
$$

This means a fixed preconditioning of the problem associated with $H_{k_{\max }}$ - with preconditioning from the right in (B) and from the left in (A). Again, linear convergence can be shown under the assumptions made in Sections 3.1 and 3.2 . 
(III) Once $k>k_{\max }$ is reached, one may also consider restricting the update loop to indices

$$
i=k-k_{\max }, \ldots, k \text { (moving window) } .
$$

For update (A), such a variant seems to be hard to interpret. For update (B), however, the update loop c) in Algorithm (B) can be solved to yield

$$
\text { a) } z_{k}=H_{0} A \Delta_{k}+\sum_{i=0}^{k-1} \gamma_{i k} \cdot\left(\Delta_{i+1}-\left(1-t_{i}\right) \Delta_{i}\right)
$$

with factors

$$
\text { b) } \gamma_{i k}:=\frac{q_{i}^{*} q_{k}}{t_{i} q_{i}^{*} q_{i}}
$$

Note that the corresponding factors $\gamma_{i k}$ in Algorithm (A) would contain $\tilde{z}_{i}$. Obviously, the moving window variant in Algorithm (B) means replacing the above sum by its most recent iterative contributions. Such a variant might seem reasonable in view of the superlinear convergence properties of secant methods. However, it is unclear whether such a variant converges at all.

Each of the above restricted storage versions was implemented and tested on several examples. It turns out that all the window variants are not competitive with variant $(I)$. Therefore, only $(I)$ will be studied in Section 5 . 


\section{Numerical Experiments}

On the basis of the above derivation, the following storage restricted algorithms are compared here:
$\mathbf{G B}\left(k_{\max }\right)$ :
Update $(A)$ with line search $(a)$, Broyden's "good"
method, restricted storage version $(I)$.
$\mathbf{B B}\left(k_{\max }\right)$ :
Update $(B)$ with line search $(b)$, Broyden's "bad"
method, restricted storage version $(I)$.
GMRES-L $\left(k_{\max }\right)$ : Program GMRES $(k)$ [13] with left preconditioning.
GMRES-R $\left(k_{\max }\right)$ : As above, but with the usual right preconditioning.

Any other variants of GB or BB are not included here, since their performance was not competitive with the two versions above. This excludes both window variants (II) and (III) of Section 4 and the different line searches $t_{k} \neq \tau_{k}$ for GB. The distinction of left and right preconditioning for GMRES has been made deliberately, since GB may be understood as some successively refined left preconditioner, whereas BB may be interpreted as some successively refined right preconditioner - which can be seen in the matrices $\widetilde{E}_{k}$ for GB and $\widehat{E}_{k}$ for BB.

Recall from Section 4 that $\mathrm{BB}\left(k_{\max }\right)$ requires about twice the array storage as the other 3 codes. Moreover, the GB code and the GMRES codes supply the residual vector only, if explicitly wanted. If the successive iterates $x_{k}$ are explicitly wanted (say, within an adaptive code or a nonlinear code [4]), then both GMRES codes need some modification, which in GMRES-R includes an additional preconditioned system solve per each iteration. Throughout the present section, only the rather simple preconditioning

$$
H_{0}=D^{-1}, \quad D:=\operatorname{diag}\left(a_{11}, \ldots, a_{n n}\right),
$$

is chosen. In a PDE context, this preconditioner takes care of the elliptic part (cf. [5]) - the rest must be taken care of by the rank-1 updates. A detailed study of different preconditioning techniques in a PDE setting will be given elsewhere.

Our test examples arise from convection-diffusion problems in 2-D of the following type:
a) $-\varepsilon \Delta u+\beta \cdot \nabla u=f$ on $\Omega \subset \mathbb{R}^{2}$,
b) $\left.u\right|_{\Gamma_{0}}=u_{0},\left.\frac{\partial u}{\partial n}\right|_{\Gamma_{1}}=0$ on $\partial \Omega=\Gamma_{0} \cup \Gamma_{1}, \Gamma_{0} \cap \Gamma_{1}=\emptyset$. 
In order to solve this problem, streamline upwind discretization with anisotropic adaptive grid refinement due to KorNHUBER/ROITZSCH [12]) is used.

\section{Example 1. Circular layer problem}

As a first special case of (5.1), we study a problem with a circular layer. For this, we set $\varepsilon=10^{-5}, f=0$ and $\beta=(y,-x)$. The domain $\Omega$ is $(0,1) \times(0,1) \backslash \Gamma_{0}$ with $\Gamma_{0}=\{(x, y): x=0.5, y \leq 0.5\}$. On the inflow boundary, we prescribe

$$
u_{0}(x, y)=\left\{\begin{array}{ll}
0 & \text { if } y>0.3 \\
1 & \text { if } y \leq 0.3
\end{array},(x, y) \in \Gamma_{0} .\right.
$$

In Fig. 5.1, the underlying grid with $n=4238$ is shown. Starting point $x_{0}$ is the interpolated solution on a coarser grid.

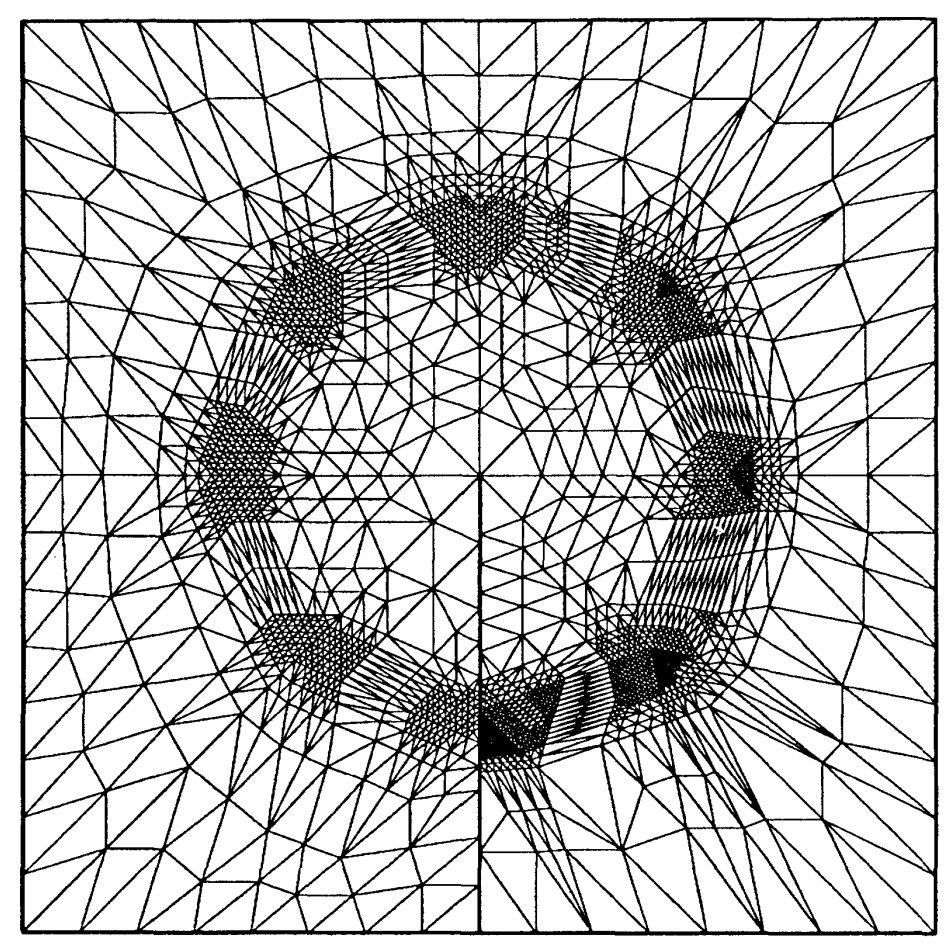

Figure 5.1: Anisotropic grid for Example 1, due to [12].

With only diagonal preconditioning, the $\mathrm{BB}$ code fails to solve the problem within $n$ steps (nearly constant residual norm throughout the iteration). First, 
the behavior of GMRES with $k_{\max } \leq 10$ has been studied (Fig. 5.2 and 5.3), which led to the selection of GMRES-R(10) as best version. This version has been compared with $\mathrm{GB}(10)$ - see Fig. 5.4. To measure the error norms, the final iterate of a $\mathrm{GB}(10)$ run with required relative accuracy $\varepsilon=10^{-8}$ in (4.3) has been taken as an estimate of the exact solution. Unlike the illustrative example in Section 3.3, the estimated error in $\mathrm{GB}(10)$ behaves only qualitatively as the true error - compare Fig. 5.5. Asymptotically, true and estimated error exhibit the same behavior, apart from oscillations caused by the $k_{\max }$-restriction.

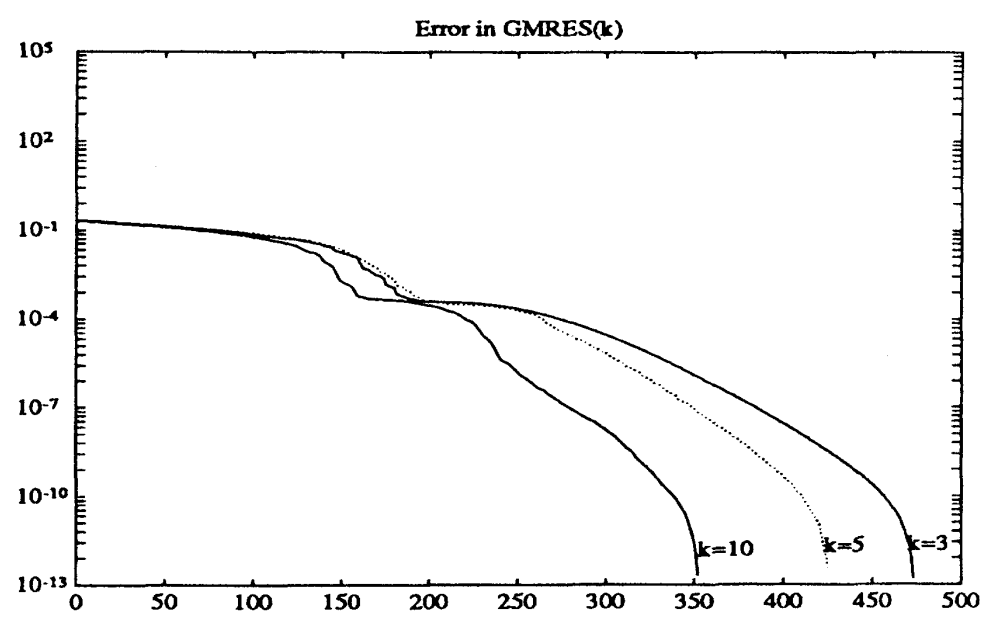

Figure 5.2: Comparison of 3 GMRES versions with right preconditioning in Example 1.

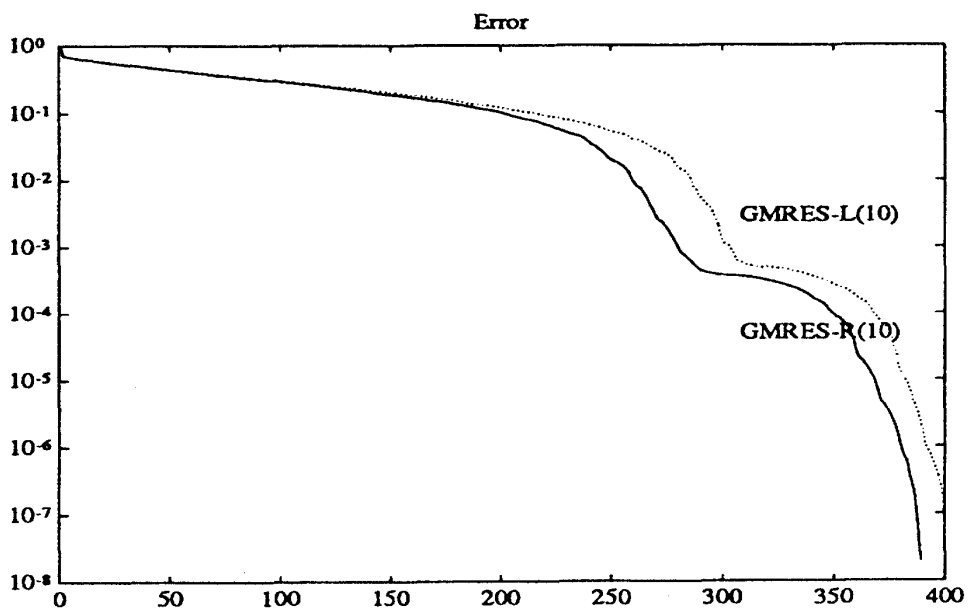

Figure 5.3: Comparison of left and right preconditioning in Example 1. 


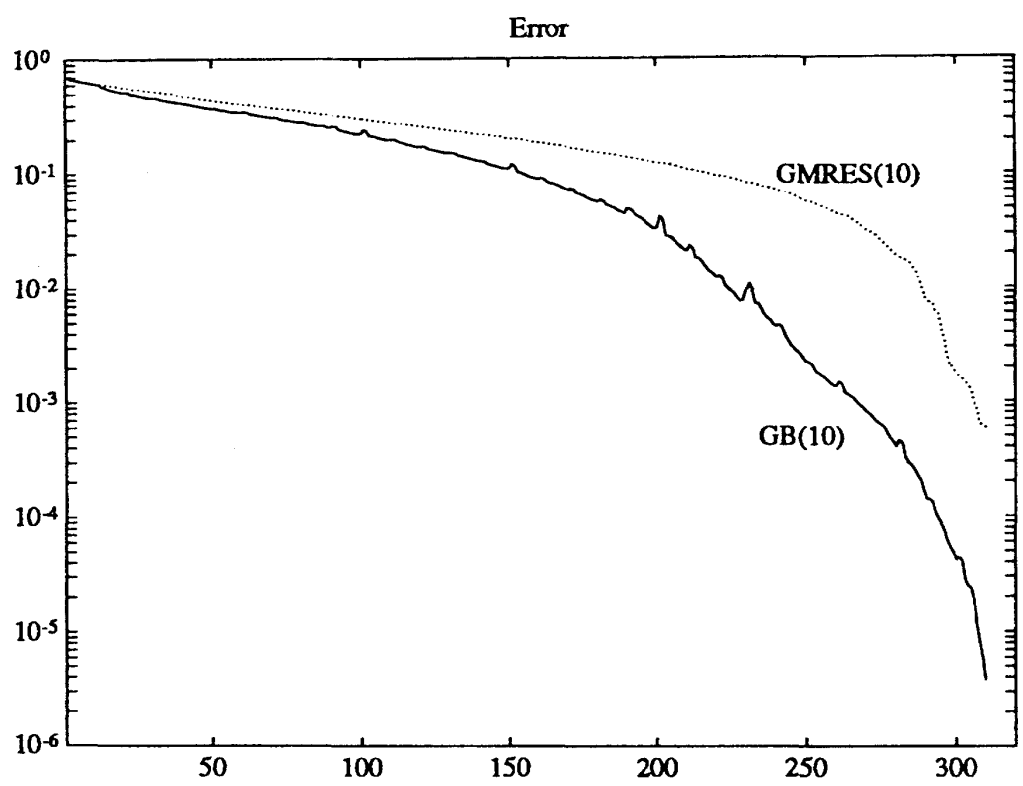

Figure 5.4: Comparison of error for GMRES and GB in Example 1.

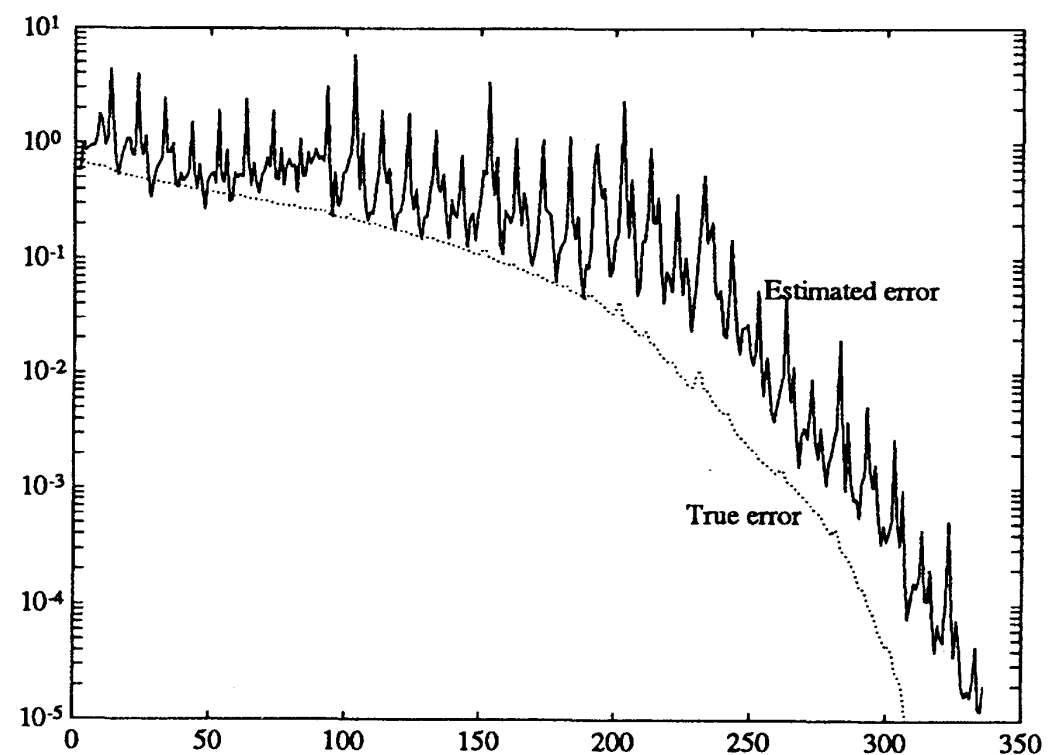

Figure 5.5: Comparison of true and estimated error in $\mathrm{GB}(10)$ in Example 1. 


\section{Example 2. Straight interior layer problem}

The second test case was the convection-diffusion equation (5.1) on $\Omega=(0,1) \times$ $(0,1)$ with a straight interior layer. To obtain this, we set $\varepsilon=10^{-6}, f=0$ and $\beta=(1.0 ; 0.5)$. The inflow boundary $\Gamma_{0}$ is given by $\Gamma_{0}=\{(x, y) \in \partial \Omega$ : $\max (x, y)<1\}$. We prescribe the boundary condition

$$
u_{0}(x, y)=\left\{\begin{array}{ll}
0 & \text { if } y>0.3 \\
1 & \text { if } y \leq 0.3
\end{array} \quad, \quad(x, y) \in \Gamma_{0}\right.
$$

In Fig. 5.6, the final grid with $n=2874$ is shown.

The behavior of the true error with diagonal preconditioning during the iteration is shown in Fig. 5.7. Once more, as in Example 1, GB appears to be the best solver. Note that the behavior in case $k_{\max }=5$ is typical also for other choices of $k_{\max }$.

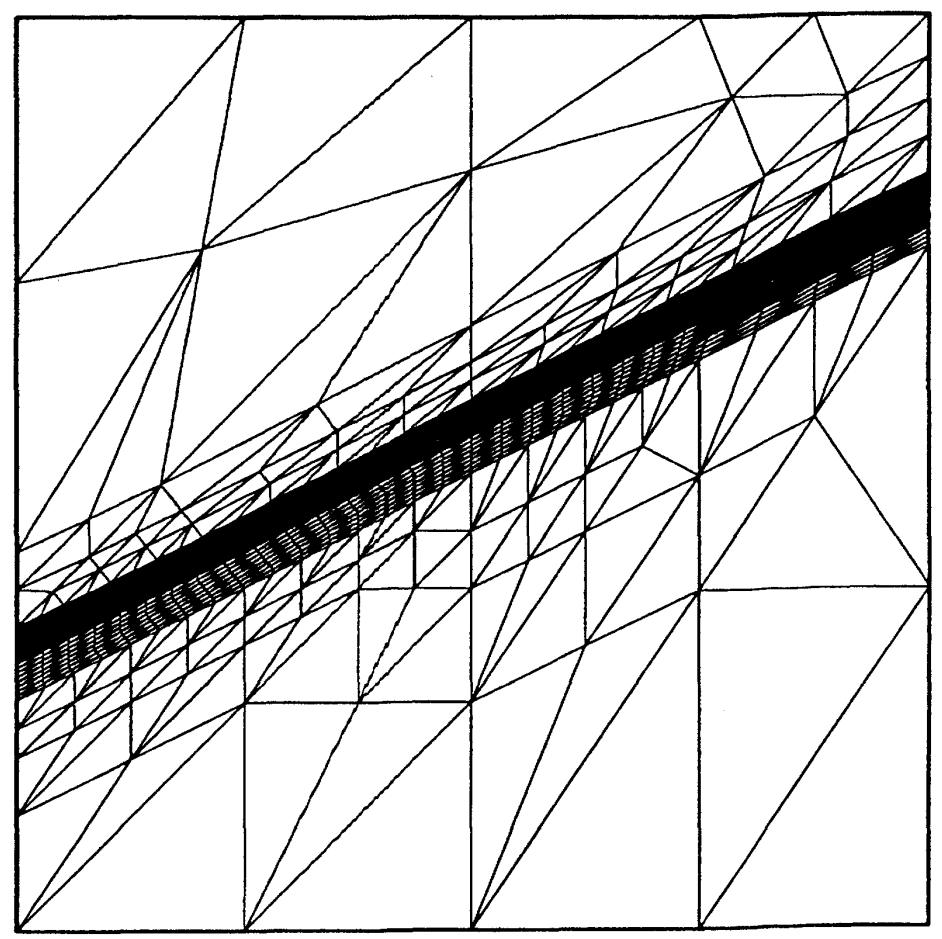

Figure 5.6: Anisotropic grid for Example 2, due to [12]. 


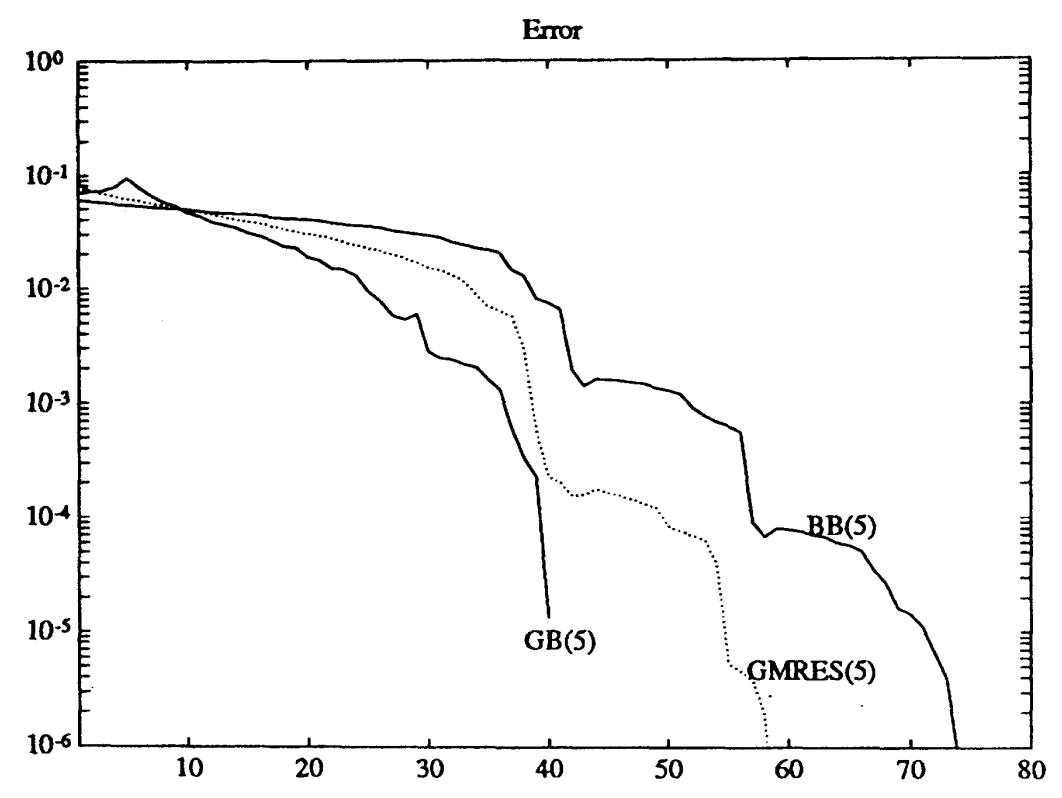

Figure 5.7: Comparison of error in $\mathrm{GB}(5), \mathrm{GMRES}(5)$ and $\mathrm{BB}(5)$ in Example 2 . 


\section{Conclusion}

Two variants of secant methods based on Broyden's "good" and "bad" rank-1 updates have been studied. It turned out to be important that each update technique is combined with its associated line search. In comparison with GMRES, the up to now bad reputation of secant methods for linear problems is certainly not justified, if a reasonable preconditioning is at hand. Especially, the "good" Broyden variant appeared to be the more competitive, the larger the system dimension was. This observation is backed not only by the given examples, but also by further more extensive tests. In the context of multilevel discretizations of PDEs, the derived secant methods seem to have the structural advantage that the arising inner products can be especially adapted to the underlying PDE problem. 


\section{References}

[1] C. G. Broyden: A class of methods for solving nonlinear simultaneous equations. Math. Comp., 19, pp. 577-593 (1965).

[2] C. G. Broyden, J. E. Dennis, and J. J. Moré: On the local and superlinear convergence of quasi-Newton methods. J. Inst. Math. Appl., 12, pp. 223245 (1973).

[3] J. E. Dennis and R. B. Schnabel: Numerical methods for unconstrained optimization and nonlinear equations. Prentice-Hall, Englewood Cliffs, NJ. (1983).

[4] P. Deuflhard: Global inexact Newton methods for very large scale nonlinear problems. Preprint SC 90-2, Konrad-Zuse-Zentrum Berlin (1990) and SIAM J. Sci. Stat. Comput., to appear.

[5] P. Deuflhard, P. Leinen and H. Yserentant: Concepts of an adaptive hierarchical finite element code. IMPACT of Computing in Science and Engineering, 1, pp. 3-35, (1989).

[6] T. Eirola and O. Nevanlinna: Accelerating with rank-one updates. Linear Algebra Appl., 121, pp. 511-520 (1989).

[7] R. Fletcher: Practical methods of optimization. Vol. 1: Unconstrained optimization. John Wiley \& Sons, New York (1980).

[8] D. M. Gay and R. B. Schnabel: Solving systems of nonlinear equations by Broyden's method with projected updates. In: Nonlinear Programming 3. O. L. Mangasarian, R. R. Meyer, and S. M. Robinson (eds.), Academic Press, New York, pp. 245-281 (1978).

[9] D. M. Gay: Some convergence properties of Broyden's method. SIAM J. Numer. Anal., 16, pp. 623-630 (1979).

[10] R. R. Gerber and F. T. Luk: A generalized Broyden's method for solving simultaneous linear equations. SIAM J. Numer. Anal., 18, pp. 882-890 (1981).

[11] M. R. Hestenes and E. Stiefel: Methods of conjugate gradients for solving linear systems. J. Res. Natl. Bur. Stand., 49, pp. 409-436 (1952). 
[12] R. Kornhuber and R. Roitzsch: On adaptive grid refinement in the presence of internal or boundary layers. IMPACT of Computing in Science and Engineering, 2, pp. 40-72 (1990).

[13] Y. Saad and M. H. Schultz: GMRES: A generalized minimal residual algorithm for solving nonsymmetric linear systems. SIAM J. Sci. Stat. Comput., 7, pp. 856-869 (1986).

[14] C. Vuik: A comparison of some GMRES-like methods. Technical Report, Delft University of Technology, Delft (1990). 
